\title{
MECHANISMS IN ENDOCRINOLOGY
}

\section{Effect of long-term exposure to air pollution on type 2 diabetes mellitus risk: a systemic review and meta-analysis of cohort studies}

\author{
Bin Wang ${ }^{1,2}$, Donghua $\mathrm{Xu}^{3}$, Zhaohai Jing ${ }^{1}$, Dawei Liư ${ }^{4}$, Shengli Yan ${ }^{1}$ and \\ Yangang Wang' \\ 'Department of Endocrinology, The Affiliated Hospital of Medical College, Qingdao University, Qingdao 266003, \\ China, ${ }^{2}$ Essencemed Clinic, Weifang 261000, China, ${ }^{3}$ Department of Rheumatology, First Affiliated Hospital of \\ Nanjing Medical University, Nanjing 210029, China and ${ }^{4}$ Department of Urinary Surgery, Southwest Hospital \\ Affiliated to Third Military Medical University, Chongqing 400038, China
}

Correspondence should be addressed to Y Wang or B Wang Email

nlwang@126.com or robin.wangqy@gmail.com

\section{Abstract}

Objective: To assess the effect of long-term exposure to air pollution on type 2 diabetes risk, a meta-analysis of prospective cohort studies was performed.

Methods: Literature search was conducted with Pubmed, Embase, and Web of Science for prospective cohort studies investigating the association of type 2 diabetes risk with increments in particulate matter (PM, diameter $<2.5 \mu \mathrm{m}$ (PM2.5) or $<10 \mu \mathrm{m}(\mathrm{PM} 10))$ or nitrogen dioxide $\left(\mathrm{NO}_{2}\right)$. We used a random-effects model to calculate the overall relative risk (RR) with $95 \% \mathrm{Cl}$.

Results: Of 808 identified articles, ten cohort studies were finally included, which involved a total of 2371907 participants and 21095 incident cases of type 2 diabetes. Elevated risk of type 2 diabetes was significantly associated with long-term exposures to high levels of PM2.5 ( $\left.R R=1.28,95 \% \mathrm{Cl} 1.06-1.55, P=0.009, l^{2}=83.5 \%\right), \mathrm{PM} 10$ (RR=1.15, 95\% Cl 1.02-1.30, $\left.P=0.022, l^{2}=0 \%\right)$, and $\mathrm{NO}_{2}\left(\mathrm{RR}=1.12,95 \% \mathrm{Cl} 1.02-1.23, P=0.015, l^{2}=63.5 \%\right)$. When using standardized risk estimates, the RRs of type 2 diabetes were significant for increments in concentrations of PM2.5 $\left(1.39\right.$ per $10 \mu \mathrm{g} / \mathrm{m}^{3}$ increment, $95 \% \mathrm{Cl}$ 1.14-1.68, $P=0.001), \mathrm{PM} 10$ (1.34 per $10 \mu \mathrm{g} / \mathrm{m}^{3}$ increment, $\left.95 \% \mathrm{Cl} 1.22-1.47, P<0.001\right)$, and NO $2\left(1.11 \mathrm{per} 10 \mu \mathrm{g} / \mathrm{m}^{3}\right.$ increment, $95 \% \mathrm{Cl} 1.07-1.16, P<0.001)$. No obvious evidence of publication bias was observed.

Conclusion: Long-term exposure to high levels of main air pollutants is significantly associated with elevated risk of type 2 diabetes mellitus.

\section{Introduction}

Type 2 diabetes mellitus is now a common disease in the world, and its prevalence is still increasing obviously in recent years $(1,2)$. The International Diabetes Federation reported that diabetes mellitus affected at least 366 million people worldwide in 2011 , and that number was expected to reach 566 million by the year 2030 (3). Though the increasing incidence of type 2 diabetes may be a result of increasing obesity, other potential risk factors have been proposed, such as air pollution $(1,2,4)$. In recent years, several major epidemiological studies have showed that air pollution is obviously associated with increased risks of cardiovascular disease, lung cancer, and natural-cause mortality $(5,6,7,8,9)$. However, the effect of air pollution on type 2 diabetes risk has not been clearly described. Several studies have assessed the association between long-term exposure to air pollution and risk of type 2 (c) 2014 European Society of Endocrinology Printed in Great Britain
Published by Bioscientifica Ltd. 
diabetes, but the association remains unclear owing to the conflicting results $(10,11,12,13,14)$. To assess the effect of long-term exposure to air pollution on type 2 diabetes risk, a meta-analysis of prospective cohort studies was performed according to the standard guideline for conducting and reporting meta-analyses of observational studies. In this meta-analysis, we focused on the associations of type 2 diabetes with increments in the concentrations of particulate matter (PM, diameter $<2.5 \mu \mathrm{m}$ (PM2.5) or $<10 \mu \mathrm{m}$ (PM10)) or nitrogen dioxide $\left(\mathrm{NO}_{2}\right)$. This study is registered at International Prospective Register of Systematic Reviews (PROSPERO), number CRD42014009081.

\section{Methods}

\section{Search strategy}

The literature search was conducted with Pubmed, Embase, and Web of Science for prospective cohort studies assessing the associations of type 2 diabetes risk with increments in PM2.5, PM10, or $\mathrm{NO}_{2}$. We also searched abstracts from the 2012 and 2013 meetings of the American Diabetes Association and the European Association for the Study of Diabetes for unpublished studies. The search time was from their commencements to February 16, 2014, and there was no language restriction. The last literature search was updated on June 162014. The following keywords were used: 'type 2 diabetes', 'diabetes mellitus', 'insulin resistance', 'air pollution', 'particulate matter', 'PM2.5', 'PM10', and ' $\mathrm{NO}_{2}$ '. Hand searching of selected journals and checking of bibliographies in relevant published reviews or articles were also performed to supplement the electronic searches.

\section{Study selection}

The inclusion criteria were as follows: i) prospective cohort studies; ii) estimated the effect of long-term exposure to air pollution, including PM2.5, PM10, and $\mathrm{NO}_{2}$, on risk of type 2 diabetes; iii) the outcome was the development of type 2 diabetes at least 3 years after follow-up; iv) type 2 diabetes was defined with an oral glucose tolerance test or fasting plasma glucose concentration, or both, according to the National Diabetes Data Group, WHO criteria, countrywide guidelines, or specified local criteria; v) reported relative risks (RR) with 95\% CIs for type 2 diabetes according to the concentrations of air pollutants. The exclusion criteria were retrospective cohort, case-control, cross-sectional, and time-series studies.
In addition, studies without usable data or low quality were also excluded. For multiple reports from the same cohort study, only the article with the longest follow-up for identical outcomes was included.

\section{Data extraction and quality assessment}

Data were extracted independently by two investigators and conflicts were adjudicated by a third investigator. Data extraction using a standardized form included a full description of the study characteristics: first author, publication year, country, study design, baseline characteristics, exposure type, data type, definition of diabetes, time of follow-up, number of participants, events of type 2 diabetes, adjustments used within analyses, and adjusted RRs with 95\% CIs for diabetes risk. The maximally adjusted risk estimates were used in the meta-analysis. All pollutant concentrations were converted, if necessary, to $\mu \mathrm{g} / \mathrm{m}^{3}$. We contacted authors for additional data or clarification where needed.

The quality of included studies was assessed using the Newcastle Ottawa Scale as recommended by the Cochrane Non-Randomized Studies Methods Working Group (15). This scale awards a maximum of nine stars to each study: four stars for the adequate selection of cohort participants, two stars for comparability of cohort participants on the basis of the design and analysis, and three stars for the adequate ascertainment of outcomes. Quality was assigned as A or excellent with 7-9 stars, B or good with 4-6 stars, and C or suboptimal with 0-3 stars. Only studies with excellent or good quality were finally included into the analysis.

\section{Statistical analysis}

The overall RRs with 95\% CIs were used to assess the effect of air pollution on type 2 diabetes risk. As Chen et al.'s study identified the linearity for the relationship between PM2.5 and diabetes, we assumed a linear relation between exposures and outcome, and RRs were further expressed for a standardized increment in pollutant concentration of per $10 \mu \mathrm{g} / \mathrm{m}^{3}$ for PM2.5, PM10, and $\mathrm{NO}_{2}$ (13). Standardized risk estimates were calculated for each study using the following formula: $\mathrm{RR}_{\text {(standardized) }}=$ $\mathrm{RR}_{\text {(original) }}^{\text {increment(10)/increment(original) }}$ (8). The overall RRs with 95\% CIs were calculated using a random-effects model for all analyses (16). The significance of the pooled RR was determined by the $Z$ test, and a $P$ value of $<0.05$ was considered significant. Statistical heterogeneity across the studies was calculated by the $I^{2}$ statistic to quantify 
inconsistencies between studies (17). $I^{2}$ values of $25 \%$ or less, $50 \%$, and $75 \%$ or more represent low, moderate, and high heterogeneity respectively. Subgroup analysis was further performed by gender (males and females) and data type (per $10 \mu \mathrm{g} / \mathrm{m}^{3}$ or interquartile range (IQR) increment). To assess the potential for publication bias, we visually inspected funnel plots. We also added the Egger regression test to test the symmetry of funnel plot (18). In addition, trim and fill method was also used to simulate those studies that may be missing from the literature, and estimated the pooled RR after adding those 'missing' studies (19). Statistical analyses were performed using Stata 12.0 (StataCorp, College Station, TX, USA). Statistical significance was taken as two-sided $P<0.05$.

\section{Results}

\section{Literature search and study characteristics}

The abstracts of 808 articles were assessed, and 42 studies underwent in-depth review, with 33 studies not fulfilling the inclusion criteria. One article reported two individual cohort studies, and was extracted as two individual studies (14). Thus, ten prospective cohort studies from nine articles were finally included into the meta-analysis (10, $11,12,13,14,20,21,22,23)$. Table 1 shows the main characteristics of those ten cohort studies included in the meta-analysis (Table 1). Those ten cohort studies involved a total of 2371907 participants and 21095 incident cases of type 2 diabetes $(10,11,12,13,14,20,21,22,23)$. Among those ten cohort studies, nine studies reported incident new cases of type 2 diabetes $(10,11,13,14,20$, $21,22,23)$, while the left one reported events of deaths coded as diabetes (12). There were three studies from USA $(10,14)$, three studies from Canada $(12,13,20)$, two studies from Germany $(21,22)$, one from Denmark (11), and one from Switzerland (24) (Table 1). The time of follow-up of those ten cohort studies ranged from 6 to 16 years (Table 1). All studies reported adjusted estimates, but the adjusted factors were various and different from each other (Table 1). There were five cohort studies on PM2.5 $(10,12,13,14)$, six cohort studies on $\mathrm{NO}_{2}(10,11,20,21$, $22,23)$, and four cohort studies on PM10 (14, 21, 23) respectively (Table 1 ). According to the quality criteria, there were seven cohort studies with A level quality (10, $11,13,14,21,23)$, and the other three studies with B level quality $(12,20,22)$ (Table 1). Among those nine cohort studies, five studies provided data on incident cases of confirmed type 2 diabetes $(10,14,21,22)$, while the other five studies made no distinction between type 2 diabetes and type 1 diabetes $(11,12,13,20,23)$. Because the vast majority of those patients with diabetes were type 2 diabetes, the associations from those four studies likely described an effect of air pollution on risk of type 2 diabetes, and they were included into the meta-analysis. In the sensitivity analysis, those five studies were further excluded to get a more precise assessment on the effects of air pollutants on type 2 diabetes risk. Among those ten cohort studies, the average concentrations of PM2.5, PM10, and $\mathrm{NO}_{2}$ were $15.2,34.1$, and $24.6 \mu \mathrm{g} / \mathrm{m}^{3}$ respectively (Fig. 1).

\section{PM2.5 and type 2 diabetes mellitus}

Meta-analysis of those five studies $(10,12,13,14)$ on PM2.5 showed that long-term exposure to high levels of PM2.5 was significantly associated with elevated risk of type 2 diabetes (per $10 \mu \mathrm{g} / \mathrm{m}^{3}$ or IQR increment $\mathrm{RR}=1.28$, 95\% CI $1.06-1.55, P=0.009, I^{2}=83.5 \%$; standardized $\mathrm{RR}=1.39,95 \%$ CI $1.14-1.68, \quad P=0.001, \quad I^{2}=86.3 \%$; Fig. 2). After excluding Brook et al.'s study, long-term exposure to high levels of PM2.5 was significantly associated with elevated risk of type 2 diabetes (per $10 \mu \mathrm{g} / \mathrm{m}^{3}$ or IQR increment $\mathrm{RR}=1.13,95 \%$ CI $1.05-1.23$, $P=0.001, \quad I^{2}=0 \% ;$ standardized $\mathrm{RR}=1.35, \quad 95 \%$ CI $\left.1.05-1.75, P=0.020, I^{2}=81.1 \%\right)$. In addition, the pooled risk estimates were not significantly altered in the sensitivity analysis performed by omitting single study in turns. After excluding those two studies $(12,13)$ making no distinction between type 2 diabetes and type 1 diabetes, long-term exposure to high levels of PM2.5 was still significantly associated with elevated risk of type 2 diabetes (per $10 \mu \mathrm{g} / \mathrm{m}^{3}$ or IQR increment $\mathrm{RR}=1.24$, 95\% CI 1.05-1.48, $P=0.014, I^{2}=0 \%$ ).

Subgroup analysis by gender showed that long-term exposure to high levels of PM2.5 was associated with elevated risk of type 2 diabetes in females (per $10 \mu \mathrm{g} / \mathrm{m}^{3}$ or IQR increment $\mathrm{RR}=1.28$, 95\% CI 1.10-1.50, $P=0.001$, $I^{2}=67.1 \%$ ), but not in males (per $10 \mu \mathrm{g} / \mathrm{m}^{3}$ or IQR increment $\mathrm{RR}=1.30,95 \%$ CI $0.95-1.77, P=0.103$, $I^{2}=90.4 \%$ ). Meta-analysis of studies reporting data of per IQR increment showed an obvious association between long-term exposure to high levels of PM2.5 and risk of type 2 diabetes (per IQR increment $\mathrm{RR}=1.25,95 \%$ CI 1.04-1.49, $P=0.014, I^{2}=0 \%$ ), but meta-analysis of studies reporting data on per $10 \mu \mathrm{g} / \mathrm{m}^{3}$ increment failed to identify an obvious association (per $10 \mu \mathrm{g} / \mathrm{m}^{3}$ increment $\mathrm{RR}=1.27,95 \%$ CI $0.97-1.66, P=0.077, I^{2}=91.4 \%$ ).

There was no obvious risk of publication bias in the funnel plot for the association between PM2.5 and type 2 


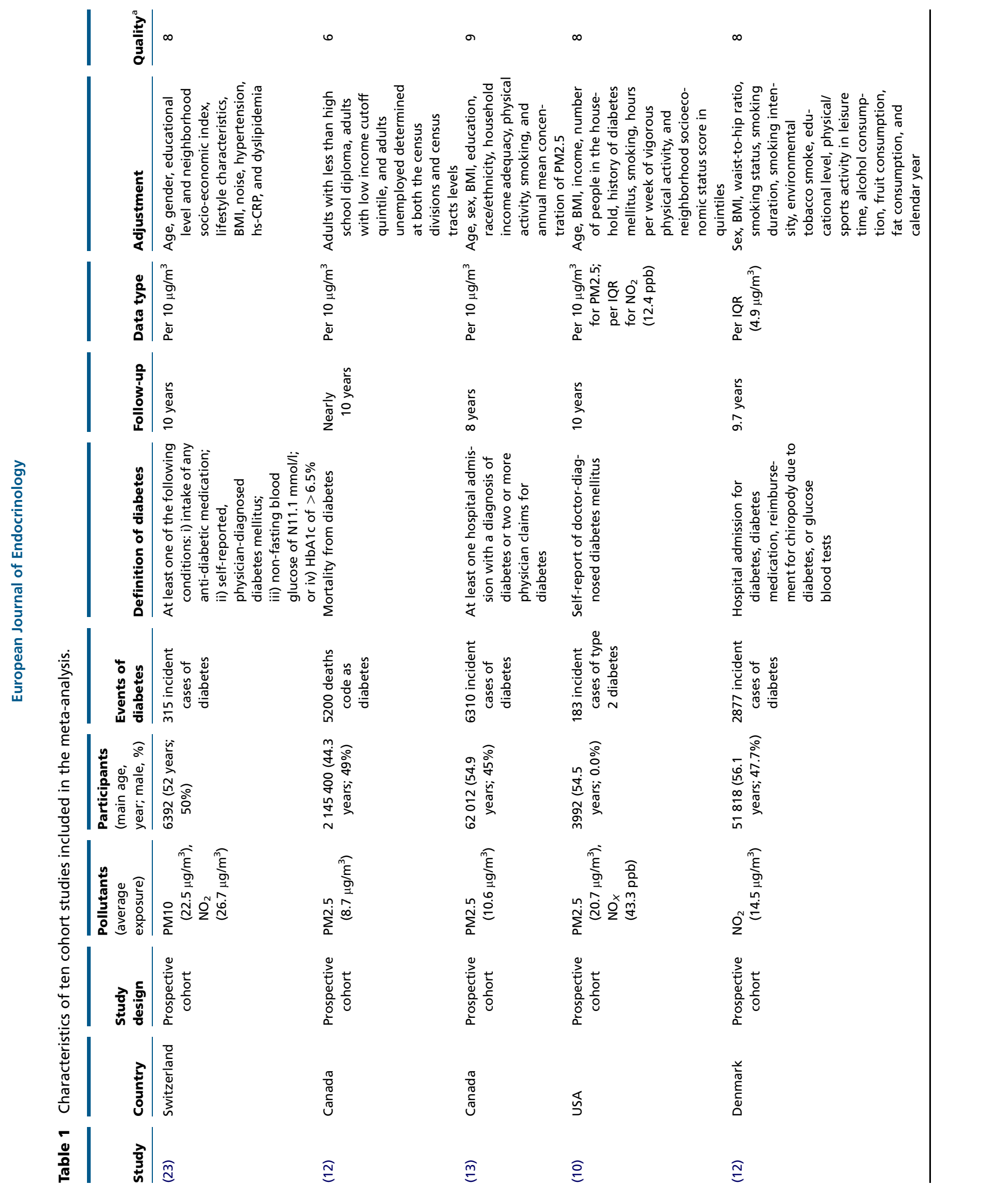




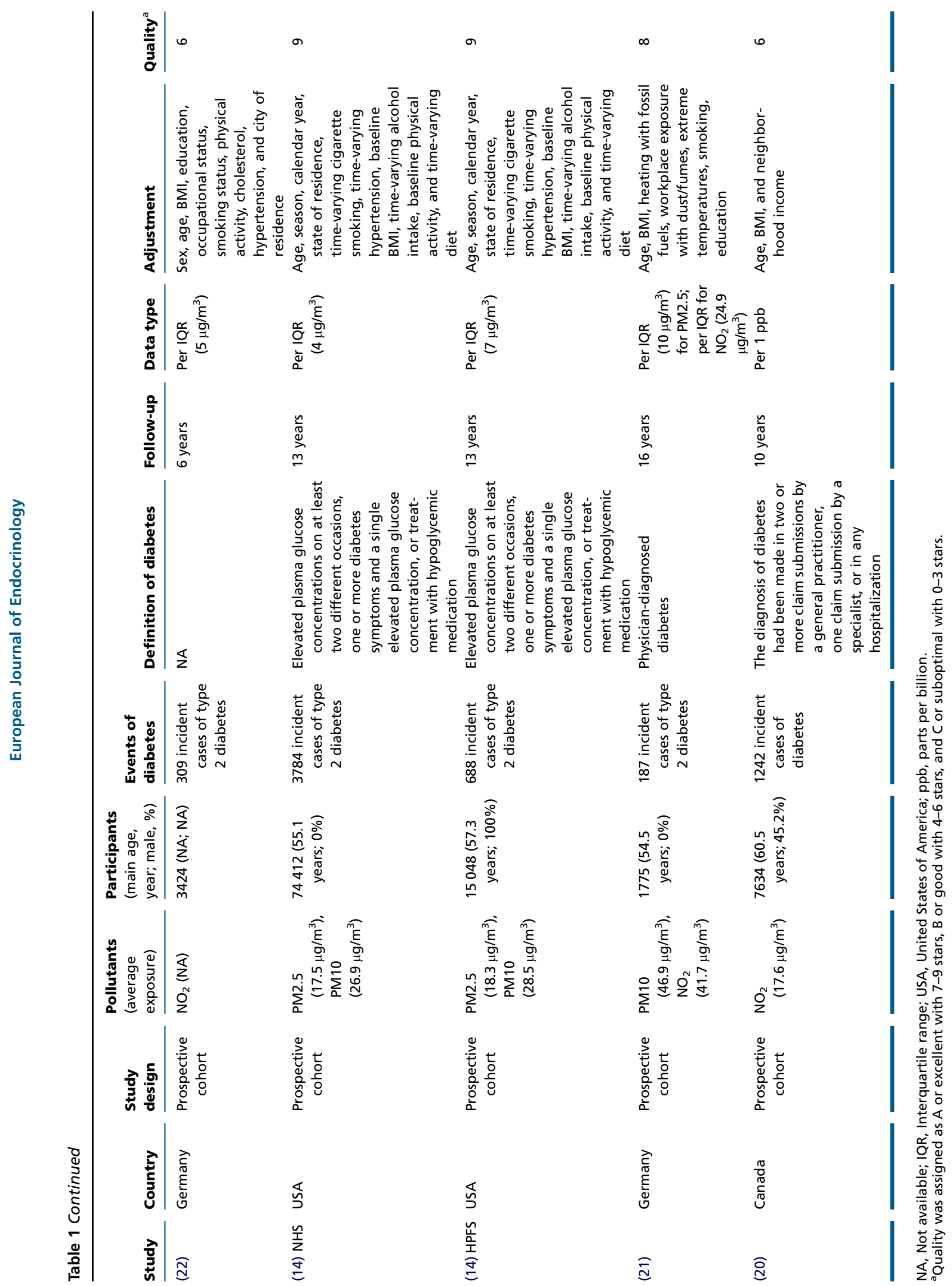


Potentially relevant studies identified through literature search $(n=808)$

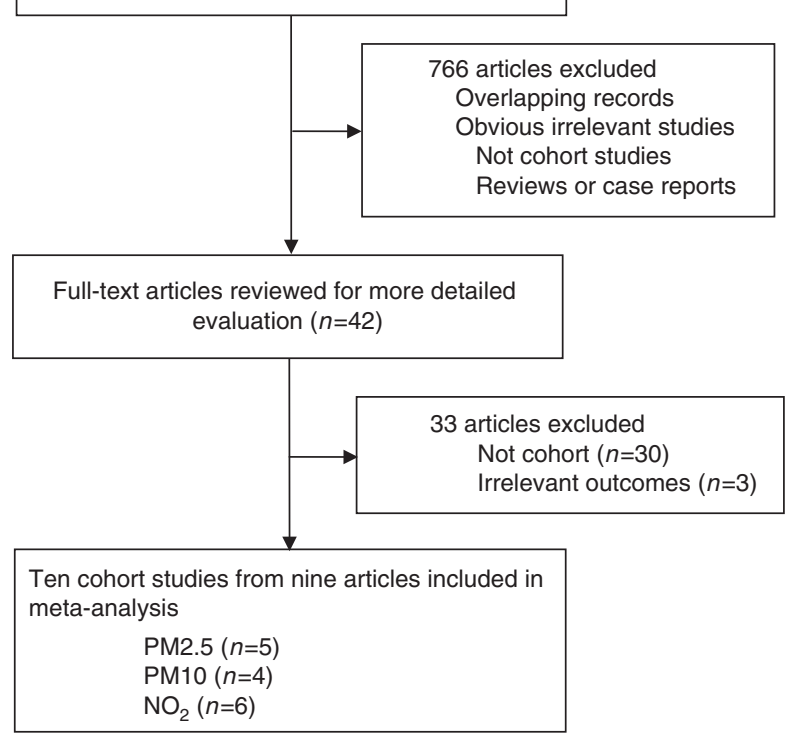

\section{Figure 1}

Flow chart of study selection in the meta-analysis.

diabetes, and the $P$ value for Egger's test was 0.98 . When using the Trim and Fill method, one possible 'missing' study was added and long-term exposure to high levels of PM2.5 was still significantly associated with elevated risk of type 2 diabetes (per $10 \mu \mathrm{g} / \mathrm{m}^{3}$ or IQR increment $\mathrm{RR}=1.26,95 \%$ CI $1.05-1.50, P=0.011)$.

\section{$\mathrm{NO}_{2}$ and type 2 diabetes mellitus}

Meta-analysis of those six studies $(10,11,20,21,22,23)$ on $\mathrm{NO}_{2}$ showed that long-term exposure to high levels of $\mathrm{NO}_{2}$ was significantly associated with elevated risk of type 2 diabetes (per IQR increment $\mathrm{RR}=1.12$, 95\% CI $1.02-1.23, P=0.015, I^{2}=63.5 \%$; standardized $\mathrm{RR}=1.11$, 95\% CI 1.07-1.16, $P<0.001, I^{2}=43.6 \%$; Fig. 3). In addition, the pooled risk estimates were not significantly altered in the sensitivity analysis performed by omitting single study in turns. After excluding those three studies $(11,20,23)$ making no distinction between type 2 diabetes and type 1 diabetes, long-term exposure to high levels of $\mathrm{NO}_{2}$ was still significantly associated with elevated risk of type 2 diabetes (per IQR increment $\mathrm{RR}=1.17$, 95\% CI 1.06-1.29, $\left.P=0.001, I^{2}=19.5 \%\right)$.

Subgroup analysis by gender showed that long-term exposure to high levels of $\mathrm{NO}_{2}$ was associated with elevated risk of type 2 diabetes in females (per IQR increment $\mathrm{RR}=1.09,95 \%$ CI $1.02-1.15, P=0.006$, $I^{2}=48.2 \%$ ), but not in males (per IQR increment $\mathrm{RR}=1.03,95 \%$ CI $\left.0.96-1.10, P=0.414, I^{2}=72.0 \%\right)$.

There was no obvious risk of publication bias in the funnel plot for the association between $\mathrm{NO}_{2}$ and type 2 diabetes, and the $P$ value for Egger's test was 0.11 . When using the Trim and Fill method, three possible 'missing' studies were added, and long-term exposure to high levels of $\mathrm{NO}_{2}$ was still significantly associated with elevated risk of type 2 diabetes (standardized $\mathrm{RR}=1.09$, 95\% CI 1.04-1.14, $P<0.001)$.

\section{PM10 and type 2 diabetes mellitus}

Meta-analysis of those four studies $(14,21,23)$ on PM10 showed that long-term exposure to high levels of PM10 was significantly associated with elevated risk of type 2 diabetes (per IQR increase $\mathrm{RR}=1.15,95 \%$ CI 1.02-1.30, $P=0.022, I^{2}=0 \%$; standardized $\mathrm{RR}=1.34$, 95\% CI 1.22 1.47, $P<0.001, I^{2}=0 \%$; Fig. 4). In sensitivity analysis by excluding the studies one by one, with the exception of NHS study by Puett et al. (14), the pooled RR for per IQR increment was not significant (per IQR increment $\mathrm{RR}=1.22$, 95\% CI 0.95-1.55, $P=0.112, I^{2}=0 \%$, but the standardized RR was still significant (standardized $\mathrm{RR}=1.36,95 \%$ CI $\left.1.18-1.57, P<0.001, I^{2}=0 \%\right)$.

Subgroup analysis by gender showed that the association between long-term exposure to high levels of PM10 and type 2 diabetes was marginally significant in females (per IQR increment $\mathrm{RR}=1.09$, 95\% CI 1.02-1.15,

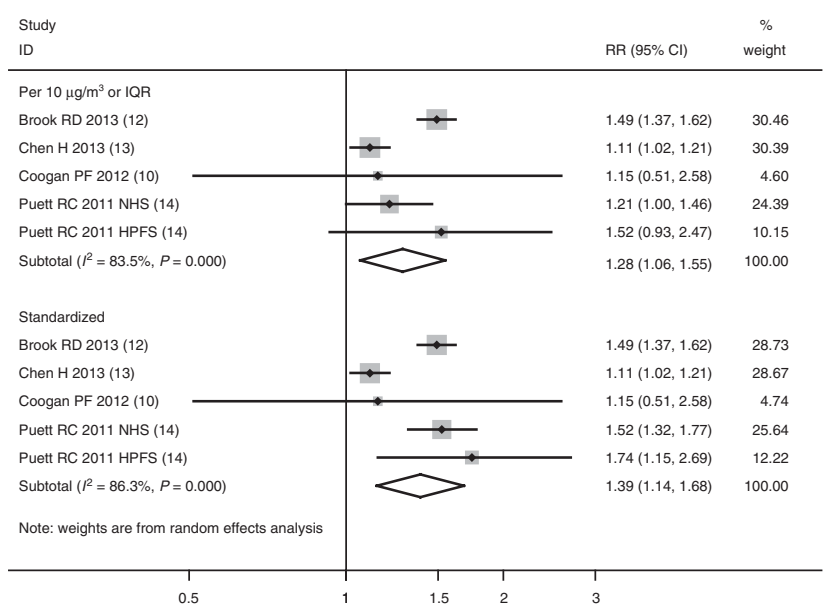

\section{Figure 2}

Association between long-term exposure to high level of PM2.5 and type 2 diabetes risk. 


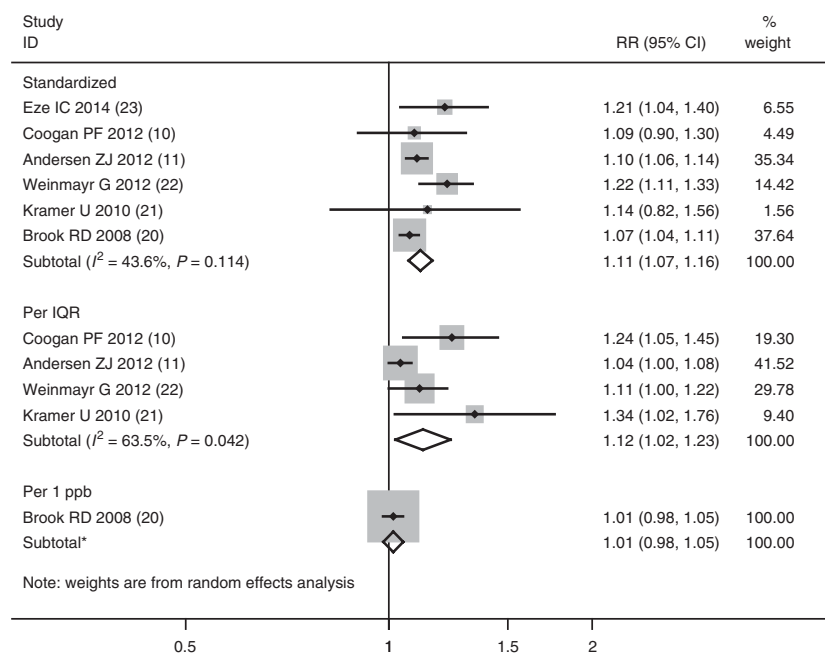

\section{Figure 3}

Association between long-term exposure to high level of $\mathrm{NO}_{2}$ and type 2 diabetes risk. $* I^{2}$ and $P$ values are not available as there was only one study and heterogeneity could not be analysed.

$P=0.006$ ), but not in males (per IQR increment $\mathrm{RR}=1.03$, 95\% CI 0.96-1.10, $P=0.414)$.

There was no obvious risk of publication bias in the funnel plot for the association between PM10 and type 2 diabetes, and the $P$ value for Egger's test was 0.76 . When using the Trim and Fill method, no possible 'missing' study was added.

\section{Discussion}

Previous studies published to assess the association between long-term exposure to air pollution and type 2 diabetes risk reported conflicting results $(10,11,12,13,14$, $20,21,22,23)$. The conflicting results may result from the low statistical power derived in a single study. Metaanalysis is a statistical method that focuses on contrasting and combining results from different studies and provides a more powerful estimate of the true effect size. Thus, to provide a more powerful estimate of the effect of longterm exposure to air pollution on type 2 diabetes risk, a meta-analysis of prospective cohort studies was performed in the study. To the best of our knowledge, the metaanalysis is the first to assess the quality and magnitude of the association between long-term exposure to air pollution and type 2 diabetes risk. The findings from the meta-analysis suggested that incident type 2 diabetes was significantly associated with long-term exposures to high levels of PM2.5, PM10, and $\mathrm{NO}_{2}$ (Figs 2, 3 and 4). Pooled results of standardized risk estimates also found the obvious associations of type 2 diabetes risk with longterm exposures to PM2.5, PM10, and $\mathrm{NO}_{2}$. In addition, sensitivity analyses further identified the obvious associations mentioned earlier. Thus, the meta-analysis suggests that long-term exposure to high levels of main air pollutants is significantly associated with increased risk of type 2 diabetes.

Subgroup analyses by gender showed that incident type 2 diabetes was significantly associated with long-term exposures to high levels of PM2.5 or $\mathrm{NO}_{2}$ in females, but not in males. The findings indicated that the effect of longterm exposure to air pollution on type 2 diabetes risk may be more profound in females than in males. The gender-specific difference in the effects of long-term exposure to air pollution on type 2 diabetes risk may relate to the differences in the biologic susceptibility to type 2 diabetes between males and females. In addition, it is also possible that the gender-specific difference may be related to the exposure assessment error, as male participants tend to be more mobile compared with female participants (4). However, considering the limited number of cohort studies on males, the findings from males are still not robust, and the effects of long-term exposure to air pollution on type 2 diabetes risk in males need further studies.

Though the risk of publication bias was not observed in the meta-analysis, the statistical power from Egger's test was limited, because there were only five studies evaluating PM2.5, six studies evaluating $\mathrm{NO}_{2}$, and four studies evaluating PM10 respectively (Table 1 ). However, the findings after adding possible 'missing' studies through the Trim and Fill method suggested that incident type 2

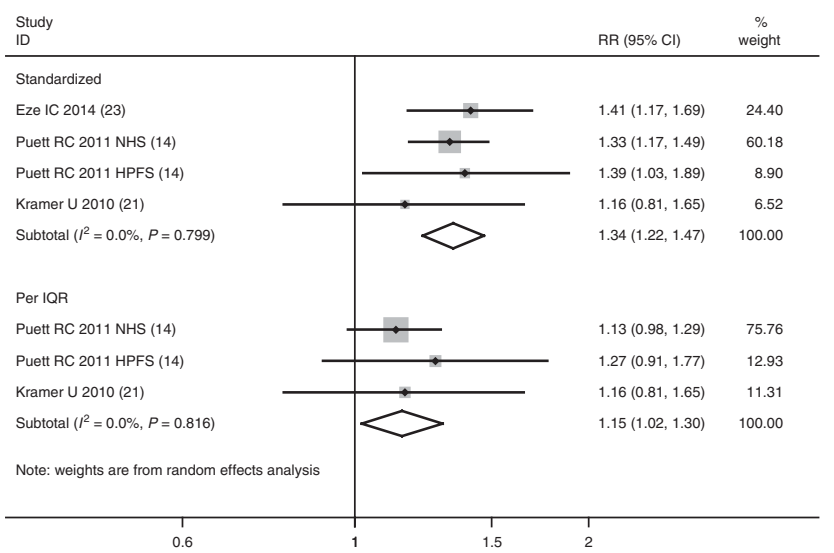

Figure 4

Association between long-term exposure to high level of PM10 and type 2 diabetes risk. 
diabetes was still significantly associated with long-term exposures to high levels of PM2.5, PM10, and $\mathrm{NO}_{2}$, which indicated that publication bias had little influence on the pooled estimates in the meta-analysis and that proved the creditability of the pooled results in the meta-analysis.

The adverse effect of long-term exposure to air pollution on type 2 diabetes risk is of much importance given the extraordinary confluence of high levels of air pollutants in urbanized environments, especially in rapidly urbanizing countries $(23,25,26)$. Air pollution in China is a significant public health burden, especially in spring and winter $(26,27)$. The pooled results of standardized risk estimates in the present meta-analysis suggest an obvious association of type 2 diabetes risk with long-term exposure to PM2.5 (Fig. 2). Compared with the average concentration of PM2.5 in the five cohort studies, the mean annual average of PM2.5 in China is much higher, and it is no doubt that people from China will suffer much higher risk of type 2 diabetes. Indeed, the overall prevalence of diabetes in China has experienced a rapid increase during the past decade (from 5.5 to $11.6 \%$ ) $(24,28,29)$, and it is much higher than that of worldwide (6.4\%) (3), which indirectly proves the rationality of the hypothesis mentioned earlier. Given the increasing burden of air pollution and its continuous and omnipresent nature, even a small adverse effect on health can represent an enormous public health issue and it should deserve rapid and positive response in public health policy. The findings from the meta-analysis provide a strong evidence for the obviously adverse effect of air pollution on type 2 diabetes mellitus; improving air quality is necessary in developing countries, which can lead to significant public health benefits. Currently, there are no trials designed to assess whether effective interventions that improve air quality are associated with a decreased incidence of type 2 diabetes. Further trials are needed to determine the effects of interventions aiming to improve air quality on risk of type 2 diabetes.

The adverse effects of air pollution on cardiovascular disease, lung cancer, and natural-cause mortality have been well established in several major epidemiological studies $(5,6,7,8,9)$. The findings from the meta-analysis further add the evidence for the adverse effect of air pollution on risk of type 2 diabetes mellitus. In fact, the influence of air pollution on risk of type 2 diabetes may further result in an increased risk of cardiovascular disease, because diabetes and insulin resistance have fundamental roles in the pathophysiology of cardiovascular disease (4). In addition, there are also many studies showing the obvious associations of air pollutants with diabetes-related mortality, which further indicate the adverse effects of air pollution on diabetes $(30,31,32,33)$. The obvious association of long-term exposures to air pollution with diabetes-related mortality has also been identified in two prospective cohort studies $(12,34)$.

Several possible mechanisms for the adverse effect of air pollution on type 2 diabetes mellitus reported herein have been suggested in recent studies. Insulin resistance is a main potential mechanism explaining the associations mentioned earlier. Both experimental and epidemiologic studies suggest that environmental exposures to air pollutants can increase the risk of insulin resistance, which may lead to an obvious link between air pollution and type 2 diabetes mellitus $(35,36,37)$. Inflammation is another potential mechanism explaining the associations reported in this meta-analysis. There is high possibility for the activation of the inflammatory pathway and oxidative stress pathway by particulate matter $(38,39$, 40). Previous studies also suggest that PM2.5 can recruit inflammatory cells via CC chemokine receptor 2-dependent mechanism, which is a known inflammatory mechanism in the pathogenesis underlying the association between air pollution and type 2 diabetes $(35,41)$. In addition, previous experimental studies also show that tumor necrosis factor- $\alpha$, interleukin- 6 , and leptin levels are also elevated on exposure to PM2.5, which suggests that exposure to ambient PM2.5 can be associated with elevated proinflammatory biomarkers and inflammatory responses $(37,39,40)$. It is clear that further data from both experimental and epidemiologic studies are needed to provide more insights into the adverse effect of air pollution on type 2 diabetes risk.

Several limitations of the meta-analysis should be considered. First, there was lack of individual participant data. Some studies used the mean concentration of air pollutants, while other studies used peak concentration of air pollutant. The variables used in the adjusted risk estimates were also different among those included cohort studies, which could result in the high heterogeneity among those studies. A meta-analysis of individual participant data is needed, which may decrease the influence of heterogeneity among those studies, and could provide a more precise assessment on the effects of air pollution on type 2 diabetes risk. Second, all cohort studies included in the meta-analysis were done in developed countries, and there was lack of data from developing countries. However, the data from developing countries may be more concerning because those regions are affected most by air pollution and may have the greatest potential to improve health by improving air 
quality. Larger cohort studies, especially from developing countries, are needed to provide a more precise assessment of the adverse effects of long-term exposure to air pollution on type 2 diabetes risk. Finally, meta-analysis of observational studies has limitations with inherent biases. The limitations of observational studies included the problem of residual confounding factors, which could also extend to the meta-analyses of observational studies and may cause some possible bias.

In conclusion, the study provides a strong evidence for the adverse effect of air pollution on type 2 diabetes risk, and long-term exposure to high levels of main air pollutants is significantly associated with elevated risk of type 2 diabetes mellitus. In addition, more prospective cohort studies with large numbers of participants, especially those from developing countries, are needed to provide a more precise assessment of the adverse effects of long-term exposure to air pollution on type 2 diabetes risk.

\section{Declaration of interest}

The authors declare that there is no conflict of interest that could be perceived as prejudicing the impartiality of the review.

\section{Funding}

This research did not receive any specific grant from any funding agency in the public, commercial or not-for-profit sector.

\section{Author contribution statement}

The authors have made the following declarations about their contributions: $B$ Wang and $Y$ Wang were the project leads for the main systematic review. D Xu, D Liu, S Yan, and Z Jing searched databases, assessed the study eligibility, and performed data extraction. Quality of data extraction and checking was carried out by B Wang, D Xu, and Z Jing. Statistical analysis was undertaken by B Wang and D Xu. B Wang and $Y$ Wang wrote the manuscript. All authors reviewed the manuscript and contributed to the preparation of the manuscript.

\section{Acknowledgements}

The authors thank Mr Zhang, from the Fudan University, Shanghai, China, for his kind help in statistics.

\section{References}

1 Nolan CJ, Damm P \& Prentki M. Type 2 diabetes across generations: from pathophysiology to prevention and management. Lancet 2011 378 169-181. (doi:10.1016/S0140-6736(11)60614-4)

2 Polonsky KS. The past 200 years in diabetes. New England Journal of Medicine 2012367 1332-1340. (doi:10.1056/NEJMra1110560)
3 Shaw JE, Sicree RA \& Zimmet PZ. Global estimates of the prevalence of diabetes for and 2030. Diabetes Research and Clinical Practice 201087 4-14. (doi:10.1016/j.diabres.2009.10.007)

4 Rajagopalan S \& Brook RD. Air pollution and type 2 diabetes: mechanistic insights. Diabetes 201261 3037-3045. (doi:10.2337/ db12-0190)

5 Cesaroni G, Forastiere F, Stafoggia M, Andersen ZJ, Badaloni C, Beelen R, Caracciolo B, de Faire U, Erbel R, Eriksen KT et al. Long term exposure to ambient air pollution and incidence of acute coronary events: prospective cohort study and meta-analysis in 11 European cohorts from the ESCAPE Project. BMJ 2014348 f7412. (doi:10.1136/ bmj.f7412)

6 Mustafic H, Jabre P, Caussin C, Murad MH, Escolano S, Tafflet M, Perier MC, Marijon E, Vernerey D, Empana JP et al. Main air pollutants and myocardial infarction: a systematic review and meta-analysis. Journal of the American Medical Association 2012307 713-721. (doi:10.1001/jama.2012.126)

7 Beelen R, Raaschou-Nielsen O, Stafoggia M, Andersen ZJ, Weinmayr G, Hoffmann B, Wolf K, Samoli E, Fischer P, Nieuwenhuijsen M et al. Effects of long-term exposure to air pollution on natural-cause mortality: an analysis of 22 European cohorts within the multicentre ESCAPE project. Lancet 2014383 785-795. (doi:10.1016/S01406736(13)62158-3)

8 Shah AS, Langrish JP, Nair H, McAllister DA, Hunter AL, Donaldson K, Newby DE \& Mills NL. Global association of air pollution and heart failure: a systematic review and meta-analysis. Lancet $2013 \mathbf{3 8 2}$ 1039-1048. (doi:10.1016/S0140-6736(13)60898-3)

9 Raaschou-Nielsen O, Andersen ZJ, Beelen R, Samoli E, Stafoggia M, Weinmayr G, Hoffmann B, Fischer P, Nieuwenhuijsen MJ, Brunekreef B et al. Air pollution and lung cancer incidence in 17 European cohorts: prospective analyses from the European Study of Cohorts for Air Pollution Effects (ESCAPE). Lancet Oncology 201314 813-822. (doi:10.1016/S1470-2045(13)70279-1)

10 Coogan PF, White LF, Jerrett M, Brook RD, Su JG, Seto E, Burnett R, Palmer JR \& Rosenberg L. Air pollution and incidence of hypertension and diabetes mellitus in black women living in Los Angeles. Circulation 2012125 767-772. (doi:10.1161/CIRCULATIONAHA.111.052753)

11 Andersen ZJ, Raaschou-Nielsen O, Ketzel M, Jensen SS, Hvidberg M, Loft S, Tjonneland A, Overvad K \& Sorensen M. Diabetes incidence and long-term exposure to air pollution: a cohort study. Diabetes Care 2012 35 92-98. (doi:10.2337/dc11-1155)

12 Brook RD, Cakmak S, Turner MC, Brook JR, Crouse DL, Peters PA, van Donkelaar A, Villeneuve PJ, Brion O, Jerrett $\mathrm{M}$ et al. Long-term fine particulate matter exposure and mortality from diabetes in Canada. Diabetes Care 201336 3313-3320. (doi:10.2337/dc12-2189)

13 Chen H, Burnett RT, Kwong JC, Villeneuve PJ, Goldberg MS, Brook RD, van Donkelaar A, Jerrett M, Martin RV, Brook JR et al. Risk of incident diabetes in relation to long-term exposure to fine particulate matter in Ontario, Canada. Environmental Health Perspectives 2013121 804-810. (doi:10.1289/ehp.1205958)

14 Puett RC, Hart JE, Schwartz J, Hu FB, Liese AD \& Laden F. Are particulate matter exposures associated with risk of type 2 diabetes? Environmental Health Perspectives 2011119 384-389. (doi:10.1289/ehp.1002344)

15 Wells G, Shea B, O'connell D, Peterson J, Welch V, Losos M \& Tugwell P. The Newcastle-Ottawa Scale (NOS) for assessing the quality of nonrandomised studies in meta-analyses. Ottawa Health Research Institute Web site, 2012

16 DerSimonian R \& Laird N. Meta-analysis in clinical trials. Controlled Clinical Trials 19867 177-188. (doi:10.1016/0197-2456 (86)90046-2)

17 Higgins JP, Thompson SG, Deeks JJ \& Altman DG. Measuring inconsistency in meta-analyses. BMJ 2003327 557-560. (doi:10.1136/ bmj.327.7414.557)

18 Egger M, Davey Smith G, Schneider M \& Minder C. Bias in metaanalysis detected by a simple, graphical test. BMJ 1997315 629-634. (doi:10.1136/bmj.315.7109.629) 
19 Peters JL, Sutton AJ, Jones DR, Abrams KR \& Rushton L. Performance of the trim and fill method in the presence of publication bias and between-study heterogeneity. Statistics in Medicine 200726 4544-4562. (doi:10.1002/sim.2889)

20 Brook RD, Jerrett M, Brook JR, Bard RL \& Finkelstein MM. The relationship between diabetes mellitus and traffic-related air pollution. Journal of Occupational and Environmental Medicine $2008 \mathbf{5 0}$ 32-38. (doi:10.1097/JOM.0b013e31815dba70)

21 Kramer U, Herder C, Sugiri D, Strassburger K, Schikowski T, Ranft U \& Rathmann $\mathrm{W}$. Traffic-related air pollution and incident type 2 diabetes: results from the SALIA cohort study. Environmental Health Perspectives 2010118 1273-1279. (doi:10.1289/ehp.0901689)

22 Weinmayr G, Fuks K, Hennig F, Moebus S, Nonnemacher M, Jakobs H, Mohlenkamp S, Erbel R, Jockel KH \& Hoffmann B. Industry and traffic related air pollution and diabetes type two incidence: results from a German cohort study. European Journal of Epidemiology 201227 S12.

23 Eze IC, Schaffner E, Fischer E, Schikowski T, Adam M, Imboden M, Tsai M, Carballo D, von Eckardstein A, Künzli N et al. Long-term air pollution exposure and diabetes in a population-based Swiss cohort. Environment International 2014 70C 95-105. (doi:10.1016/j.envint. 2014.05.014)

24 Xu Y, Wang L, He J, Bi Y, Li M, Wang T, Jiang Y, Dai M, Lu J, Xu M et al. Prevalence and control of diabetes in Chinese adults. Journal of the American Medical Association 2013310 948-959. (doi:10.1001/ jama.2013.168118)

25 Rich DQ, Kipen HM, Huang W, Wang G, Wang Y, Zhu P, Ohman-Strickland P, Hu M, Philipp C, Diehl SR et al. Association between changes in air pollution levels during the Beijing Olympics and biomarkers of inflammation and thrombosis in healthy young adults. Journal of the American Medical Association 2012307 2068-2078.

26 Guo Y, Li S, Tian Z, Pan X, Zhang J \& Williams G. The burden of air pollution on years of life lost in Beijing, China, 2004-08: retrospective regression analysis of daily deaths. BMJ $20133 \mathbf{3 4 7}$ f7139. (doi:10.1136/ bmj.f7139)

27 Gong P, Liang S, Carlton EJ, Jiang Q, Wu J, Wang L \& Remais JV. Urbanisation and health in China. Lancet 2012379 843-852. (doi:10. 1016/S0140-6736(11)61878-3)

$28 \mathrm{Zuo} \mathrm{H}$, Shi Z \& Hussain A. Prevalence, trends and risk factors for the diabetes epidemic in China: a systematic review and meta-analysis. Diabetes Research and Clinical Practice 2014104 63-72. (doi:10.1016/ j.diabres.2014.01.002)

29 Yang W, Lu J, Weng J, Jia W, Ji L, Xiao J, Shan Z, Liu J, Tian H, Ji Q et al. Prevalence of diabetes among men and women in China. New England Journal of Medicine 2010362 1090-1101. (doi:10.1056/ NEJMoa0908292)

30 Pearson JF, Bachireddy C, Shyamprasad S, Goldfine AB \& Brownstein JS. Association between fine particulate matter and diabetes prevalence in the U.S. Diabetes Care 201033 2196-2201. (doi:10.2337/dc10-0698)
31 Chiusolo M, Cadum E, Stafoggia M, Galassi C, Berti G, Faustini A, Bisanti L, Vigotti MA, Dessi MP, Cernigliaro A et al. Short term effects of nitrogen dioxide on mortality and susceptibility factors in ten Italian cities: the EpiAir Study. Environmental Health Perspectives 2011119 1233-1238. (doi:10.1289/ehp.1002904)

32 Ostro B, Broadwin R, Green S, Feng WY \& Lipsett M. Fine particulate air pollution and mortality in nine California counties: results from CALFINE. Environmental Health Perspectives 2006114 29-33. (doi:10.1289/ehp.8335)

33 Forastiere F, Stafoggia M, Berti G, Bisanti L, Cernigliaro A, Chiusolo M, Mallone S, Miglio R, Pandolfi P, Rognoni M et al. Particulate matter and daily mortality: a case-crossover analysis of individual effect modifiers. Epidemiology 200819 571-580. (doi:10.1097/EDE.0b013e3181761f8a)

34 Raaschou-Nielsen O, Sorensen M, Ketzel M, Hertel O, Loft S, Tjonneland A, Overvad K \& Andersen ZJ. Long-term exposure to traffic-related air pollution and diabetes-associated mortality: a cohort study. Diabetologia 201356 36-46.

35 Liu C, Xu X, Bai Y, Wang TY, Rao X, Wang A, Sun L, Ying Z, Gushchina L, Maiseyeu A et al. Air pollution-mediated susceptibility to inflammation and insulin resistance: influence of CCR2 pathways in mice. Environmental Health Perspectives 2014122 17-26.

36 Thiering E, Cyrys J, Kratzsch J, Meisinger C, Hoffmann B, Berdel D, von Berg A, Koletzko S, Bauer CP \& Heinrich J. Long-term exposure to traffic-related air pollution and insulin resistance in children: results from the GINIplus and LISAplus birth cohorts. Diabetologia 201356 1696-1704. (doi:10.1007/s00125-013-2925-x)

37 Sun Q, Yue P, Deiuliis JA, Lumeng CN, Kampfrath T, Mikolaj MB, Cai Y, Ostrowski MC, Lu B, Parthasarathy S et al. Ambient air pollution exaggerates adipose inflammation and insulin resistance in a mouse model of diet-induced obesity. Circulation 2009119 538-546. (doi:10.1161/CIRCULATIONAHA.108.799015)

38 Teichert T, Vossoughi M, Vierkotter A, Sugiri D, Schikowski T, Schulte T, Roden M, Luckhaus C, Herder C \& Kramer U. Association between traffic-related air pollution, subclinical inflammation and impaired glucose metabolism: results from the SALIA study. PLOS ONE 20138 e83042. (doi:10.1371/journal.pone.0083042)

39 O'Neil MS, Veves A, Sarnat JA, Zanobetti A, Gold DR, Economides PA, Horton ES \& Schwartz J. Air pollution and inflammation in type 2 diabetes: a mechanism for susceptibility. Occupational and Environmental Medicine 200764 373-379. (doi:10.1136/oem.2006. 030023)

40 Franchini M \& Mannucci PM. Thrombogenicity and cardiovascular effects of ambient air pollution. Blood $2011 \mathbf{1 1 8} 2405-2412$. (doi:10.1182/blood-2011-04-343111)

41 Potera C. Toxicity beyond the lung: connecting PM2.5, inflammation, and diabetes. Environmental Health Perspectives 2014122 A29. (doi:10.1289/ehp.122-A29)

Received 6 May 2014

Revised version received 1 July 2014

Accepted 8 July 2014 